\title{
The formation of giant planets in wide orbits by photoevaporation-synchronised migration
}

\author{
O. M. Guilera, ${ }^{1,2 \star ~ M . ~ M . ~ M i l l e r ~ B e r t o l a m i ~}{ }^{1} \dagger$ M. P. Ronco, ${ }^{1,2}$ \\ ${ }^{1}$ Instituto de Astrofísica de La Plata, CCT La Plata, CONICET-UNLP, Paseo del Bosque S/N, B1900FWA La Plata, Argentina \\ ${ }^{2}$ Facultad de Ciencias Astronómicas y Geofísicas, UNLP, Paseo del Bosque S/N, B1900FWA La Plata, Argentina
}

Accepted XXX. Received YYY; in original form ZZZ

\begin{abstract}
The discovery of giant planets in wide orbits represents a major challenge for planet formation theory. In the standard core accretion paradigm planets are expected to form at radial distances $\lesssim 20$ au in order to form massive cores (with masses $\gtrsim 10 \mathrm{M}_{\oplus}$ ) able to trigger the gaseous runaway growth before the dissipation of the disc. This has encouraged authors to find modifications of the standard scenario as well as alternative theories like the formation of planets by gravitational instabilities in the disc to explain the existence of giant planets in wide orbits. However, there is not yet consensus on how these systems are formed.

In this letter, we present a new natural mechanism for the formation of giant planets in wide orbits within the core accretion paradigm. If photoevaporation is considered, after a few Myr of viscous evolution a gap in the gaseous disc is opened. We found that, under particular circumstances planet migration becomes synchronised with the evolution of the gap, which results in an efficient outward planet migration. This mechanism is found to allow the formation of giant planets with masses $M_{p} \lesssim 1 M_{\text {Jup }}$ in wide stable orbits as large as $\sim 130$ au from the central star.
\end{abstract}

Key words: planets and satellites: formation - protoplanetary discs - planet-disc interactions

\section{INTRODUCTION}

The discovery of giant planets in very wide orbits by Kalas et al. (2008) and Marois et al. (2008) posed a challenge to planet formation theory. In the core accretion mechanism giant planets are expected to form at radial distances $a \lesssim 20$ au in order to form cores which are massive enough to trigger the gaseous runaway growth (with masses $M_{p} \gtrsim 10 \mathrm{M}_{\oplus}$ ) before the dissipation of the disc. While the core accretion mechanism was very successful in explaining the properties of planets and planetary systems discovered by radial velocities and transits, the explanation of giant planets in wide orbits, like those surrounding Fomalhaut and HR 8799, is difficult. Dodson-Robinson et al. (2009) performed simple numerical experiments for the formation of planets in the core accretion mechanism and concluded that even under the most favourable conditions the formation of giant planets at $a \gtrsim 35$ au is not possible. In addition, by performing N-body simulations Dodson-Robinson et al. (2009) showed that planet-planet scattering was not able to

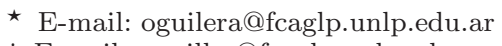

$\dagger$ E-mail: mmiller@fcaglp.unlp.edu.ar create stable wide-orbit systems such as HR 8799. They concluded that massive (gas) giant planets in wide stable orbits with $a \gtrsim 35$ au are formed by gravitational instabilities in the disc. Boss (2011) studied the formation of giant planets in wide orbits by gravitational instabilities in discs of different masses and around different possible protostars, and concluded that the formation by gravitational instabilities of gas giants with semi-major axes $a \simeq 30^{\circ} 70 \mathrm{au}$, eccentricities $e \simeq 0 \backsim 0.35$, and masses $M_{p} \simeq 1 \backsim 5 M_{\text {Jup }}$ would be possible if the disc fragments managed to survive to longer times. Later, however, Vorobyov (2013) showed that disc fragments have a low probability of survival due to inward migration of ejection mechanisms during the embedded phase of star formation. Consequently, Vorobyov (2013) concluded that the disc instability mechanism was unable to form gas giants with semi-major axes $a \lesssim 178$ au and proposed that planets with semi-major axes from a few tens to 150 au are likely to form via dynamical scattering or other mechanisms. Therefore, the question of whether disc instabilities can finally settle into stable wide orbits remains open.

Aside from the disc instability mechanism other alternatives have been discussed like the recapture of free floating planets Perets \& Kouwenhoven (2012) or modifications 
within the core accretion model. Examples of the latter are efficient outward type II migration (Crida et al. 2009), and high solid accretion rates (Lambrechts et al. 2014). During the development of our previous population synthesis work (Ronco et al. 2017) we serendipitously found that a small percentage of the total of the performed simulations presented a giant planet in an extended orbit at the end of the gaseous phase, between $40 \mathrm{au}$ and $100 \mathrm{au}$. An inspection of the results showed these protoplanets migrating outwards in synchrony with the outer boundary of the density gap opened by photoionisation. In this letter we present a detailed account of this phenomenon, which results in a natural scenario for the formation of giant planets in stable wide orbits within the framework of the core accretion paradigm of planet formation.

The letter is organised as follows, in section 2 we briefly describe the model of planet formation with emphasis in the upgrades with respect to our previous works. Then, in section 3 we describe the scenario and describe our numerical results. Finally, on section 4 we make some final statements and concluding remarks.

\section{MODEL AND INPUT PHYSICS}

We adopt an improved version of the planet formation code PlanetalP, adopted in our previous work (Ronco et al. 2017). Specifically we have incorporated a standard 1D+1D treatment to solve the vertical and radial structure of a viscous irradiated accretion disc and incorporated the type I migration rates for non-isothermal discs. In what follows we briefly describe the implementation of these changes and the most relevant properties of the model for the present work.

\subsection{The gaseous disc}

We assume a thin, axisymmetric, irradiated disc in hydrostatic equilibrium. To solve the vertical structure of the disc we follow the classical methodology as in Papaloizou \& Terquem (1999) and Alibert et al. (2005), solving for each radial bin the following structure equations

$\frac{\partial P}{\partial z}=-\rho \Omega^{2} z, \quad \frac{\partial F}{\partial z}=\frac{9}{4} \rho \nu \Omega^{2}, \quad \frac{\partial T}{\partial z}=\nabla \frac{T}{P} \frac{\partial P}{\partial z}$

where $P, \rho, F, T$ and $z$ represents the pressure, density, radiative heat flux, temperature and vertical coordinate of the disc, respectively. $\Omega$ is the Keplerian-frequency at a given radial distance, while $v=\alpha c_{s}^{2} / \Omega$ is the viscosity (Shakura \& Sunyaev 1973), being $c_{s}^{2}=P / \rho$ the square of the locally isothermal speed of the sound. We consider that heat is transported by radiation and convection according to the standard Schwarzschild criterion. The actual temperature gradient $\nabla=d \log T / d \log P$ in convective regions is computed with the help of the mixing length theory, following the approach of Kippenhahn et al. (2012). On purely radiative regions the temperature gradient $\left(\nabla_{\text {rad }}\right)$ is computed in the diffusive approximation valid for optically thick discs

$\nabla_{\mathrm{rad}}=\frac{3 \kappa \rho F}{16 \sigma \Omega^{2} z T^{4}}$

where $\sigma$ is the Stefan-Boltzmann constant and $\kappa$ is the local Rosseland mean opacity (Bell \& Lin 1994). We adopt the equation of state of an ideal diatomic gas $P=\rho k T / \mu m_{\mathrm{H}}$ where $k$ is the Boltzmann constant, $\mu=2$ is the mean molecular weight, $m_{\mathrm{H}}$ is the mass of the hydrogen atom, and the the adiabatic gradient for a diatomic gas is $\nabla_{\mathrm{ad}}=2 / 7$.

Following Papaloizou \& Terquem (1999), Alibert et al. (2005) and Migaszewski (2015) the boundary conditions at the surface of the disc $H, P_{S}=P(z=H), F_{S}=F(z=H)$, $T_{S}=T(z=H)$, and $F_{0}=F(z=0)$, are given by

$$
\begin{aligned}
P_{S} & =\frac{\Omega^{2} H \tau_{\mathrm{ab}}}{\kappa_{S}}, \quad F_{s}=\frac{3 \dot{M}_{\mathrm{st}} \Omega^{2}}{8 \pi}, \quad F_{0}=0, \\
0 & =2 \sigma\left(T_{s}^{4}+T_{\mathrm{irr}}^{4}-T_{\mathrm{b}}^{4}\right)-\frac{9 \alpha \Omega k\left(T_{s}^{4}+T_{\mathrm{irr}}^{4}\right)^{1 / 4}}{8 \kappa_{s} \mu m_{\mathrm{H}}}-F_{S},
\end{aligned}
$$

where $\tau_{\mathrm{ab}}=0.01$ is the optical depth, $T_{\mathrm{b}}=10 \mathrm{~K}$ is the background temperature, and $\dot{M}_{\mathrm{st}}$ is the equilibrium accretion rate. The temperature associated with the stellar irradiation is given by

$T_{\text {irr }}=T_{\star}\left[\frac{2}{3 \pi}\left(\frac{R_{\star}}{R}\right)^{3}+\frac{1}{2}\left(\frac{R_{\star}}{R}\right)^{2}\left(\frac{H}{R}\right)\left(\frac{d \log H}{d \log R}-1\right)\right]^{0.5}$,

where $R_{\star}=2 R_{\odot}$ and $T_{\star}=4000 \mathrm{~K}$ are the radius and effective temperature of the protostar, $R$ is the radial coordinate, and $d \log H / d \log R=9 / 7$. To solve the vertical structure of the disc we follow a numerical approach similar to the one described in Alibert et al. (2005) and Migaszewski (2015). This allows us to obtain the relation $v_{\mathrm{m}}\left(\Sigma_{\mathrm{g}}, R\right)$, where $\Sigma_{\mathrm{g}}$ is the gas surface density, required to solve the radial evolution of the disc (eq. 5) as well as other relationships which are needed to compute the planet migration rates (see next section).

The time evolution of the gas surface density is represented by a diffusion equation (Pringle 1981)

$\frac{\partial \Sigma_{\mathrm{g}}}{\partial t}=\frac{3}{R} \frac{\partial}{\partial R}\left[R^{1 / 2} \frac{\partial}{\partial R}\left(v_{\mathrm{m}} \Sigma_{\mathrm{g}} R^{1 / 2}\right)\right]+\dot{\Sigma}_{\mathrm{w}}(R)$,

where $\dot{\Sigma}_{\mathrm{W}}(R)$ represents a sink term due to the photoevaporation by the central star, which is given by (D’Angelo \& Marzari 2012)

$\dot{\Sigma}_{\mathrm{W}}(R)= \begin{cases}\dot{\Sigma}_{\mathrm{W}}^{g} \exp \left[\frac{1}{2}\left(1-\frac{R_{\mathrm{g}}}{R}\right)\right]\left(\frac{R_{\mathrm{g}}}{R}\right)^{2} & \text { if } R \leq R_{\mathrm{g}}, \\ \dot{\Sigma}_{\mathrm{W}}^{g}\left(\frac{R_{\mathrm{g}}}{R}\right)^{5 / 2} & \text { if } R>R_{\mathrm{g}},\end{cases}$

where $R_{\mathrm{g}}$ is the distance along the midplane beyond which gas at the disk surface is unbound. We take $R_{\mathrm{g}}=10 \mathrm{au}$ as it is usually assumed for solar-mass stars. The photoevaporation rate at $R_{\mathrm{g}}$ is represented by $\dot{\Sigma}_{\mathrm{w}}^{g}$ and is given by

$\dot{\Sigma}_{\mathrm{W}}^{g}=1.16 \times 10^{-11} \sqrt{f_{41}}\left(\frac{R_{\mathrm{g}}}{1 \mathrm{au}}\right)^{-1.5} \frac{M_{\odot}}{\mathrm{au}^{2} \mathrm{yr}}$,

where $f_{41}$ is the rate of EUV ionising photons emitted by the star in units of $10^{41} \mathrm{sec}^{-1}$.

\subsection{Planet growth and planet migration}

We considered the formation of a planet in the standard core accretion mechanism by the concurrent accretion of solid and gas. Initially, the core of the planet grows by the accretion of planetesimals in the oligarchic growth regime. As 

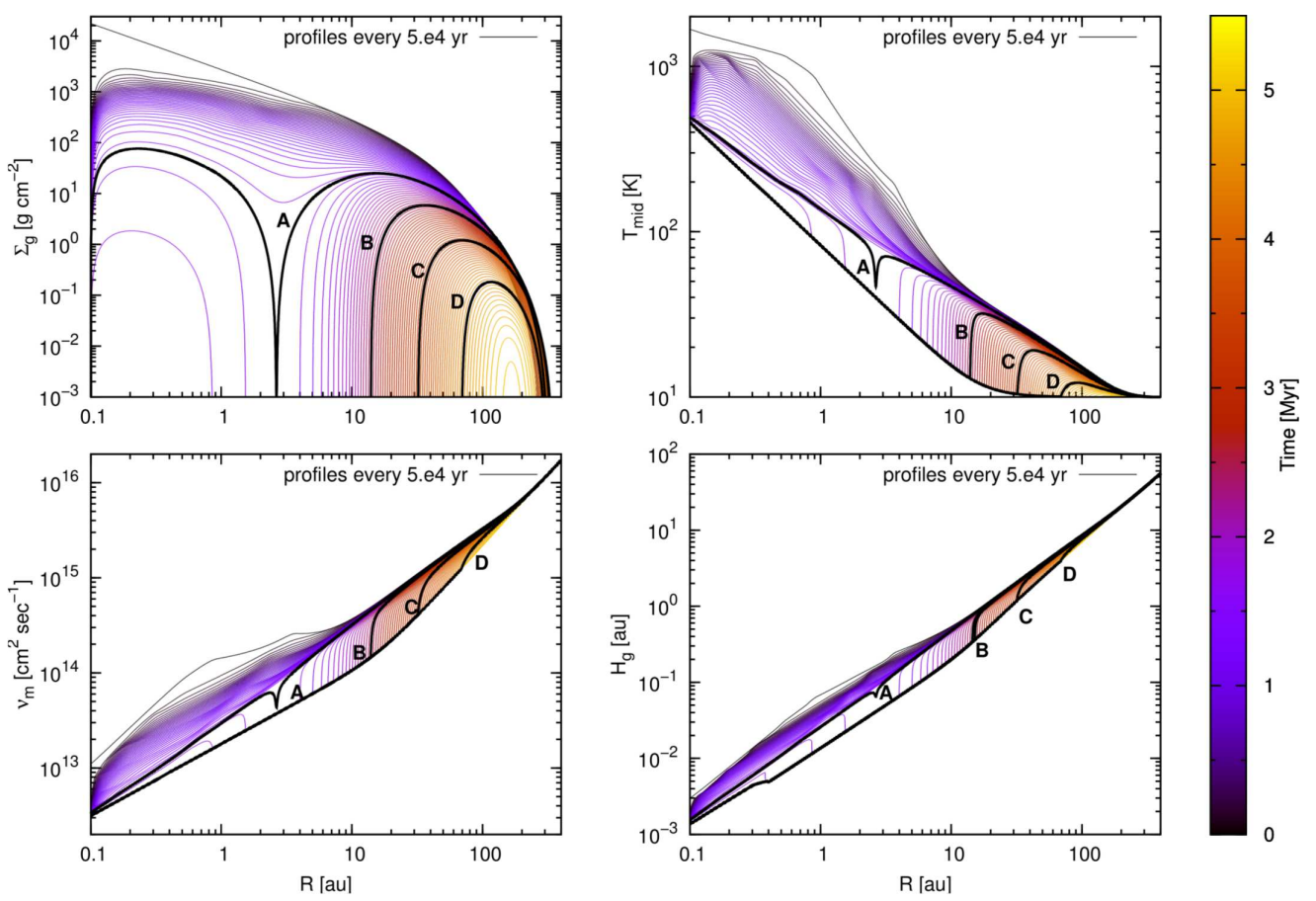

Figure 1. Time evolution of the radial profiles of the gas surface density ( $\Sigma_{\mathrm{g}}$; top left panel), the mid-plane temperature $\left(T_{\text {mid }}\right.$; top right panel), the mean viscosity $\left(v_{\mathrm{m}}\right.$; bottom left panel), and the scale height of the disc $\left(\boldsymbol{H}_{\mathrm{g}}\right.$; bottom right panel). The radial profiles A,B,C, and $\mathrm{D}$ (black thick lines) represent the structure of the disc at $\sim 1.6 \mathrm{Myr}$ (when the gap is opened due to photoevaporation), at $2.5 \mathrm{Myr}$, at $3.5 \mathrm{Myr}$, and at 4.5 Myr, respectively. The disc is dissipated at $~ 5 \mathrm{Myr}$.

the planet grows, it binds the surrounding gas, reaches a critical mass (of about a tens Earth masses) and accretes a substantial gaseous envelope. The gas accretion rate is estimated from the results of Guilera et al. (2010, 2014) following the prescriptions of Ida \& Lin (2004) and Miguel et al. (2011). We include the limitation in the gas accretion rate due to the capability of the disc to supply enough material (Mordasini et al. 2009), and the possibility that the planet opens a gap in the gaseous disc (Tanigawa \& Ikoma 2007). The population of planetesimals is considered to evolve by the drift of planetesimals due to the nebular gas including the Epstein, Stokes and quadratic regimes, and the accretion and ejection by the embryos - see Ronco et al. (2017) for details. We consider that the evolution of the eccentricities and inclinations of the planetesimal population are due to two main processes, the embryo gravitational excitations and the damping due to the nebular gas.

Of special interest for the present work is the treatment of planet migration. The interaction between the gaseous disc and the planet leads to an exchange of angular momentum producing torques that cause the planet's migration along the disc. If the planet is not able to open a gap in the disc we consider that it migrates under type I migration

$\left(\frac{d a_{\mathrm{P}}}{d t}\right)_{\operatorname{migI}}=-2 a_{\mathrm{P}} \frac{\Gamma}{L_{\mathrm{P}}}$,

where $a_{\mathrm{P}}$ is the semi-major axis of the planet, $L_{\mathrm{P}}$ is the angular momentum of the planet, and $\Gamma$ is the total torque. In this work, we incorporate the prescriptions of Paardekooper et al. $(2010,2011)$ to calculate the total torque for non-isothermal discs. When the planet is massive enough to open a gap in the disc (Crida et al. 2006), the migration of the planet switches to the known type II migration (Armitage 2007; Mordasini et al. 2009).

\section{RESULTS AND DISCUSSION}

Fig. 1 shows the evolution of gas density $\Sigma_{\mathrm{g}}$, mid-plane temperature $T_{\text {mid }}$, mean viscosity $v_{\mathrm{m}}$ and gas-disc scale-height $H_{\mathrm{g}}$ for our reference model. This model was computed for a disc with a mass of $M_{\mathrm{d}}=0.1 M_{\odot}$, a viscosity parameter $\alpha=$ $10^{-3}$, a characteristic radius of $R_{C}=39$ au, and an initial surface density profile of $\Sigma_{\mathrm{g}}(R)=\Sigma_{0}\left(R / R_{C}\right)^{-\gamma} \exp \left((R / R c)^{-(2-\gamma)}\right)$ with $\gamma=0.9$ (Andrews et al. 2010), surrounding a pre-main sequence star of $1 M_{\odot}$. As shown in Fig. 1 (curve A) photoevaporation opens a gap in the disc at $R \simeq 2.65$ au after $\sim 1.6 \mathrm{Myr}$ of evolution. Due to the strong dependence of type I migration rates with the slope of the density and temperature profiles (Paardekooper et al. 2011) the opening of the gap leads to the creation of a region of outward type I migration (ROM; blue zones in Fig. 2). As time passes, the combined effects of viscous evolution and photoevaporation widen the gap and push the ROM outwards (Fig. 1, curves $\mathrm{B}, \mathrm{C}$ and $\mathrm{D}$ ). The ROM is bounded at any time by two rings, the zero density inner boundary and the zero torque outer boundary. Note that planets migrating according to type I migration cannot cross the zero torque ring at any moment. Within the ROM, if a planet is massive enough to have an efficient type I migration then it will move outwards faster than the inner boundary does but, as it cannot cross the outer boundary it will be forced to move inside the ROM. As long as the disc is not dissipated the mass of the planet can only increase, which in most cases means more 


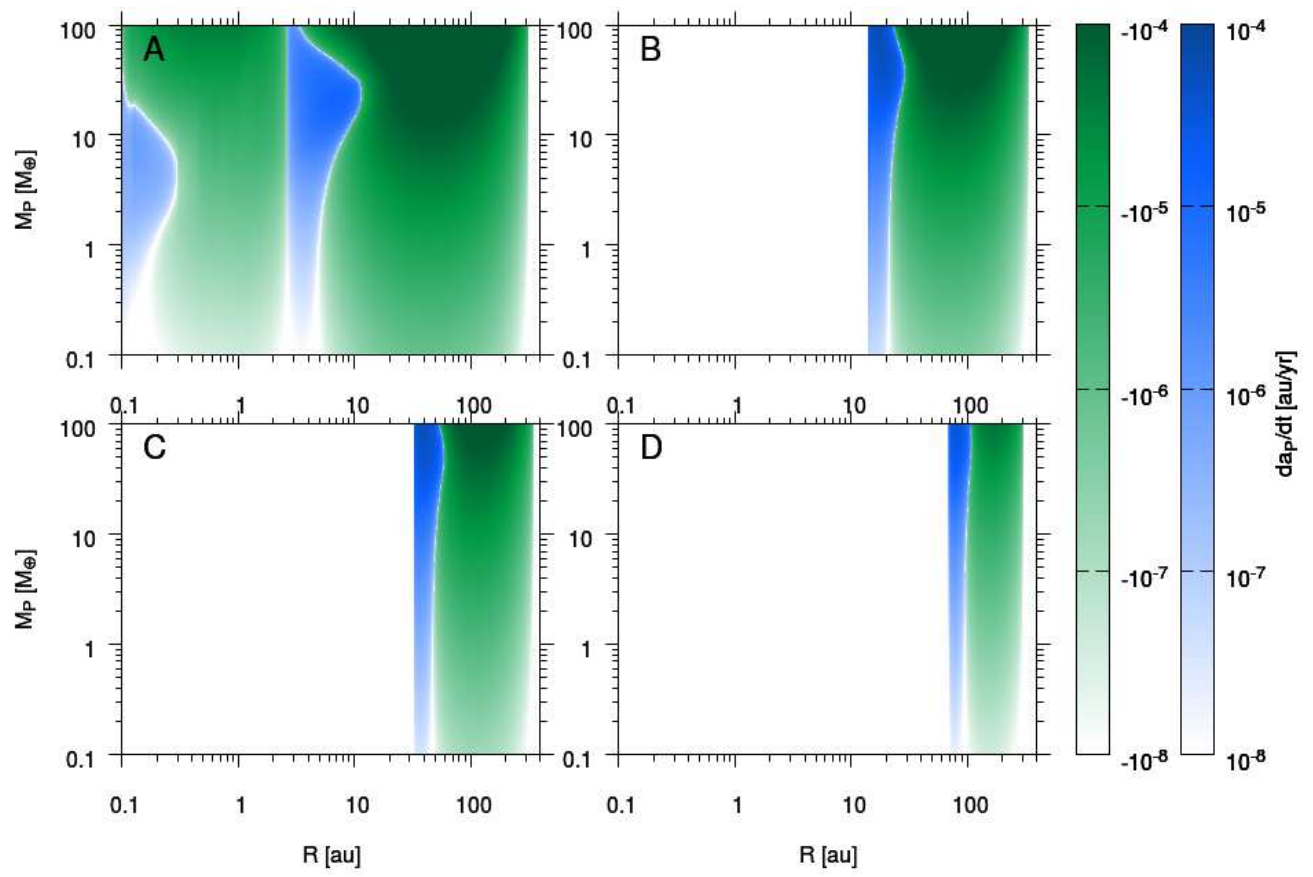

Figure 2. Migration rates for the four disc structures (A,B, C and D) shown in Fig. 1 as a function of the distance from the central star and mass of the planet at different times of the disc evolution. Panel A represents the migration map when the gap is opened in the disc. Panels B, C, and D represent the migration map at 2.5 Myr, at 3.5 Myr, and at 4.5 Myr, respectively. The blue palette represents the outward migration zone, while the green palette represents the inward migration zones.

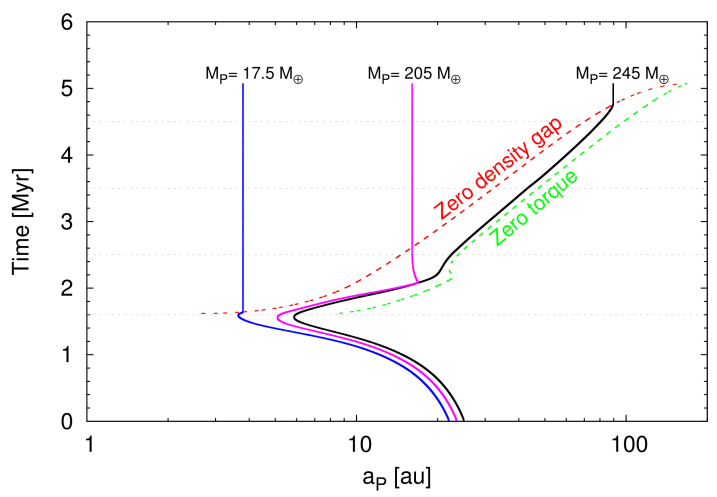

Figure 3. Evolution of the planet semi-major axis as a function of time for our reference case. The red dashed line indicates the evolution of the outer boundary of the photoevaporation gap and the green dashed line the evolution of the zero torque location in the outer part of the disc. Grey horizontal lines correspond to times A, B, C and D in Figs. 1 and 2 (from bottom to top). Different curves corresponds to protoplanets that entered the ROM with different masses and had different evolutions (see text).

efficient migration preventing the planet to lag behind the ROM. It is possible to imagine that a forming planet could be trapped in the ROM and grow as it migrates outwards synchronised with the outwards evolution of the ROM. We term these objects synchronised outwards migrating planets (SOMP). SOMPs will keep evolving outwards until the gas disc is dissipated or they become massive enough to change to a type II migration regime (Crida et al. 2006).

Fig. 3 shows the evolution of one of this SOMPs in our reference disc model (black curve, Fig. 3). The embryo, hav- ing initially a mass of $0.01 M_{\oplus}$ and located at $25 \mathrm{au}$, migrates inwards with increasing speed as the mass of the protoplanet increases by the accretion of planetesimals of $\sim 10 \mathrm{~km}$ of radius. By the time the gap is opened at $\sim 1.6 \mathrm{Myr}$ the forming planet already has a mass of $\sim 10 M_{\oplus}$ and undergoes efficient type I migration. As a consequence, as photoevaporation opens the gap, and changes the slopes of the temperature and density profiles, the direction of migration changes suddenly from a normal inward type I migration into an outward migration. After that point the planet evolves outward inside the ROM (bounded by the red and green curves in Fig. $3)$. The outward migration is finished at $\sim 4.8 \mathrm{Myr}$, when the gas disc becomes too thin. By the time migration stops the planet has increased its mass up to $M_{p} \sim 250 M_{\oplus}$ and reached a wide stable orbit at $\sim 90 \mathrm{au}$. This example shows that photoevaporation coupled to type I migration can naturally lead to the formation of massive planets in wide orbits within the core accretion framework. For a planet to become synchronised with the ROM and to evolve to very wide orbits the planet needs to reach the ROM with enough mass to have an efficient type I migration. If the mass is too small, outwards migration will be inefficient and the planet will be caught up by the zero density gap, stopping its gas accretion and migration (blue curve, Fig. 3). On the contrary, if the planet is too massive by the time it enters the ROM it might only evolves for a short time before becoming massive enough to open a gap and shift to a type II migration regime (pink curve, Fig. 3). Consequently, for the SOMP phenomenon to develop and produce planets in very wide orbit, the protoplanet needs to enter the ROM with masses in a narrow range around $10 M_{\oplus}$.

In Fig. 4 we show similar cases of photoevaporationsynchronised migration computed under different assump- 


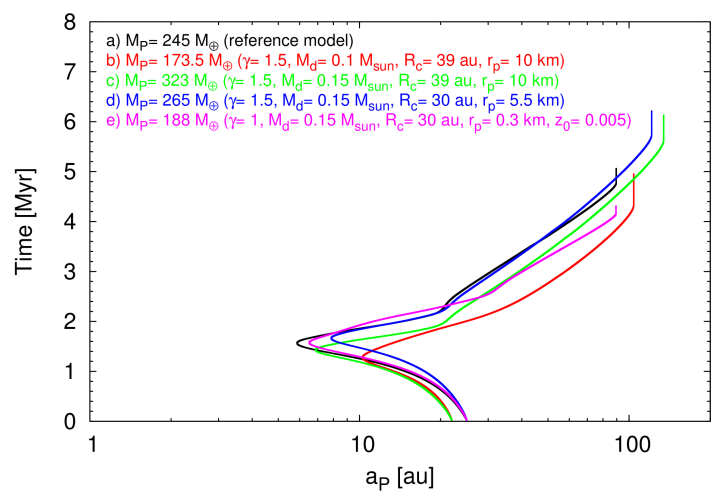

Figure 4. Evolution of the planet semi-major axes as a function of time for different SOMPs formed in different discs. $M_{P}$ represents the final masses of the planets. Quantities between brackets the parameters of the different discs. Specially interest remains in case e) where the disc have a metalicity similar to HR8799.

tions for the structure of the disc and the photoevaporation rates (for all cases we use $\alpha=10^{-3}$ ). We check the stability of the discs adopting the Toomre $Q$ parameter (Toomre 1964). As can be seen in Fig. 4 the photoevaporationsynchronised scenario is able to predict the formation of planets of $0.55 \backsim 1 M_{\mathrm{Jup}}$ as far as $90 \backsim 130$ au from the central star within the core accretion framework. In these exploratory simulations we find that most of our giant planets are formed with a high content of solids, and consequently should have smaller radii than planets formed through the disc instability mechanism. This opens the possibility to test both scenarios (Spiegel \& Burrows 2012). While the quantitative results predicted by this scenario are dependent on the disc mass, and the adopted photoevaporation (Gorti et al. 2009; Owen \& Jackson 2012) and type I migration models, the qualitative behaviour predicted here should be present in all models with an outward moving gap and a type I migration model dependent on the disc density profile.

\section{CONCLUSIONS}

We have presented a new scenario for the formation of giant planets in wide orbits within the core accretion paradigm that arises naturally from the synchronization of planet migration and disc photoevaporation. We have shown that this scenario is able to form Jupiter like-planets in wide orbits as large as $\sim 130 \mathrm{au}$. The scenario requires the mass of the protoplanet to be fine tuned at the moment it is trapped in the region of outwards type I migration, but our computations show that this scenario works in a wide region of the parameter space (Fig. 4). The fact that discs with large inner holes are more common around Herbig Ae/Be than around solar mass stars (Maaskant et al. 2013) suggests that this mechanism might be more viable to form giant planets in wide orbits around A-type stars (Kalas et al. 2008; Marois et al. 2008). Further exploration of this scenario by means of population synthesis studies is required to assess the fraction of protoplanets that undergo photoevaporation synchronised migration and, also, to establish the general properties (masses, radii and semi-major axes) predicted by this scenario. In particular, it remains to be seen if multiple planet systems like those observed in HR8799 can be formed within this picture once resonances are taken into account. We plan to explore these possibilities in future works.

\section{ACKNOWLEDGEMENTS}

We thank Yann Alibert and the anonymous referee for their suggestions to improve this work. O.M.G and M.P.R are supported by CONICET and UNLP through grants PIP0436 and G-144. M3B is partially supported by ANPCyT through grant PICT-2014-2708 and by a Return Fellowship from the Alexander von Humboldt Foundation.

\section{REFERENCES}

Alibert Y., Mordasini C., Benz W., Winisdoerffer C., 2005, A\&A, 434,343

Andrews S. M., Wilner D. J., Hughes A. M., Qi C., Dullemond C. P., 2010, ApJ, 723, 1241

Armitage P. J., 2007, ApJ, 665, 1381

Bell K. R., Lin D. N. C., 1994, ApJ, 427, 987

Boss A. P., 2011, ApJ, 731, 74

Crida A., Morbidelli A., Masset F., 2006, Icarus, 181, 587

Crida A., Masset F., Morbidelli A., 2009, ApJ, 705, L148

D’Angelo G., Marzari F., 2012, ApJ, 757, 50

Dodson-Robinson S. E., Veras D., Ford E. B., Beichman C. A., 2009, ApJ, 707, 79

Gorti U., Dullemond C. P., Hollenbach D., 2009, ApJ, 705, 1237

Guilera O. M., Brunini A., Benvenuto O. G., 2010, A\&A, 521, A50

Guilera O. M., de Elía G. C., Brunini A., Santamaría P. J., 2014, A\&A, 565, A96

Ida S., Lin D. N. C., 2004, ApJ, 604, 388

Kalas P., et al., 2008, Science, 322, 1345

Kippenhahn R., Weigert A., Weiss A., 2012, Stellar Structure and Evolution, doi:10.1007/978-3-642-30304-3.

Lambrechts M., Johansen A., Morbidelli A., 2014, A\&A, 572, A35

Maaskant K. M., et al., 2013, A\&A, 555, A64

Marois C., Macintosh B., Barman T., Zuckerman B., Song I., Patience J., Lafrenière D., Doyon R., 2008, Science, 322, 1348 Migaszewski C., 2015, MNRAS, 453, 1632

Miguel Y., Guilera O. M., Brunini A., 2011, MNRAS, 417, 314

Mordasini C., Alibert Y., Benz W., Naef D., 2009, A\&A, 501,1161

Owen J. E., Jackson A. P., 2012, MNRAS, 425, 2931

Paardekooper S.-J., Baruteau C., Crida A., Kley W., 2010, MNRAS, 401, 1950

Paardekooper S.-J., Baruteau C., Kley W., 2011, MNRAS, 410, 293

Papaloizou J. C. B., Terquem C., 1999, ApJ, 521, 823

Perets H. B., Kouwenhoven M. B. N., 2012, ApJ, 750, 83

Pringle J. E., 1981, ARA\&A, 19, 137

Ronco M. P., Guilera O. M., de Elía G. C., 2017, preprint, (arXiv: 1705.08608)

Shakura N. I., Sunyaev R. A., 1973, A\&A, 24, 337

Spiegel D. S., Burrows A., 2012, ApJ, 745, 174

Tanigawa T., Ikoma M., 2007, ApJ, 667, 557

Toomre A., 1964, ApJ, 139, 1217

Vorobyov E. I., 2013, A\&A, 552, A129

This paper has been typeset from a $\mathrm{T}_{\mathrm{E}} \mathrm{X} / \mathrm{LAT}_{\mathrm{E}} \mathrm{X}$ file prepared by the author. 